\title{
Seroprevalence for norovirus genogroups GII and GIV in captive non-human primates
}

\author{
Federica Di Profio ${ }^{1}$ | Vittorio Sarchese ${ }^{1}$ | Irene Melegari $^{1}$ | Andrea Palombieri ${ }^{1}$ | \\ Ivano Massirio $^{2}$ | Sandra Bermudez Sanchez ${ }^{1}$ | Klaus Gunther Friedrich ${ }^{3}$ | \\ Federico Coccia $^{3}$ | Fulvio Marsilio ${ }^{1}$ | Vito Martella ${ }^{4}$ | Barbara Di Martino ${ }^{1}$
}

${ }^{1}$ Faculty of Veterinary Medicine, Università degli Studi di Teramo, Teramo, Italy

${ }^{2}$ Azienda USL di Reggio Emilia, Reggio Emilia, Italy

${ }^{3}$ Fondazione Bioparco, Rome, Italy

${ }^{4}$ Faculty of Veterinary Medicine, Università Aldo Moro di Bari, Valenzano, Italy

\section{Correspondence}

Barbara Di Martino, Faculty of Veterinary Medicine, Università degli Studi di Teramo, Teramo, Italy.

Email: bdimartino@unite.it

Funding information

University of Teramo, Italy; Italian Ministry of University; Marie Skłodowska-Curie COFUNDREP-EAT, Grant/Award Number: 713714

\begin{abstract}
Noroviruses (NoVs) are a major cause of epidemic gastroenteritis in children and adults. Several pieces of evidence suggest that viruses genetically and antigenically closely related to human NoVs might infect animals, raising public health concerns about potential cross-species transmission. The natural susceptibility of non-human primates (NPHs) to human NoV infections has already been reported, but a limited amount of data is currently available. In order to start filling this gap, we screened a total of 86 serum samples of seven different species of NPHs housed at the Zoological Garden (Bioparco) of Rome (Italy), collected between 2001 and 2017, using an enzyme-linked immunosorbent assay (ELISA) based on virus-like particles (VLPs) of human GII.4 and GIV.1 NoVs. Antibodies specific for both genotypes were detected with an overall prevalence of $32.6 \%$. In detail, IgG antibodies against GII.4 NoVs were found in 18 Japanese macaques $(29.0 \%$, $18 / 62)$, a mandrill $(10.0 \%, 1 / 10)$, a white-crowned mangabey $(16.6 \%, 1 / 6)$ and in an orangutan (33.3\%, 1/3). Twelve macaques (19.3\%, 12/62), five mandrills (50.0\%, 5/10), two chimpanzees (100\%, 2/2) and a white-crowned mangabey $(16.6 \%, 1 / 6)$ showed antibodies for GIV.1 NoVs. The findings of this study confirm the natural susceptibility of captive NHPs to GII NoV infections. In addition, IgG antibodies against GIV.1 were detected, suggesting that NHPs are exposed to GIV NoVs or to antigenically related NoV strains.
\end{abstract}

\section{KEYWORDS}

genotypes GII.4 and GIV.1, IgG antibodies, non-human primates, noroviruses

\section{1 | INTRODUCTION}

Noroviruses (NoVs) are a leading cause of sporadic cases and outbreaks of acute gastroenteritis across all age groups (Patel et al., 2008). NoVs are ubiquitous, associated with $18 \%$ of diarrhoeal disease worldwide and are estimated to cause 212,000 deaths annually worldwide (Lopman, Steele, Kirkwood, \& Parashar, 2016).

NoVs are small non-enveloped viruses classified into the genus Norovirus, family Caliciviridae. The icosahedral capsid surrounds a 7.7kb positive-sense single-stranded RNA genome organized into three open reading frames (ORFs). ORF1 encodes a large polyprotein that is cleaved into many non-structural proteins. ORF2 encodes a major capsid protein (VP1) while ORF3 a small basic protein (VP2) (Green, 2013). Based on the full-length VP1 amino acid sequence, NoVs have been divided into seven genogroups (G) and at least 40 genotypes (Vinjé, 2015). Viruses belonging to GI, GII and GIV can infect humans, with GII.4 strains being the most prevalent worldwide (Green, 2013).

NoV infections have also been documented in non-human primates (NHPs) (Bok et al., 2011; Farkas, Cross et al., 2010; Farkas, Dufour, Jiang, \& Sestak, 2010; Farkas, 2016; He et al., 2017; Rockx, 
Bogers, Heeney, Van Amerongen, \& Koopmans, 2005; Sestak et al., 2012; Subekti et al., 2002; Wyatt et al., 1978). Experimental inoculation of different species of NHPs has demonstrated their susceptibility to NoVs, with active seroconversion and faecal shedding of NoV, although mainly in the absence of clinical disease (Bok et al., 2011; Rockx et al., 2005; Sestak et al., 2012; Subekti et al., 2002; Wyatt et al., 1978). Natural NoV infection in NHPs has been reported only occasionally so far. Using baculovirus-expressed VP1 of the human GI.1, GII.4 and GII.7 NoVs, IgG-specific antibodies were first detected in three Old World monkey species (mangabeys, pigtail and rhesus macaques) and in chimpanzees housed at the Yerkes National Primate Research Center (US) with rates ranging from 63.0\% to $92.0 \%$ (Jiang, McClure, Fankhauser, Monroe, \& Glass, 2004). A high prevalence (51.0\%-61.0\%) of antibodies against GI.1 and GII.5 has also been reported in a serological survey performed on a colony of rhesus macaques of the Tulane National Primate Research Center (US) (Farkas, Cross et al., 2010). Direct evidence for natural NoV infections for GI, GII and GIV NoVs has been obtained by molecular screening of 500 faecal samples from rhesus macaques with an overall prevalence of 8.2\% (Farkas, Dufour et al., 2010; Farkas, 2016). More recently, GII sequences genetically close ( $99 \%$ nucleotide identity) to the novel NoV GII.P17_GII.17 variant Kawasaki 2014 have been detected in $32.0 \%$ of stool samples collected from rhesus monkeys living in a breeding facility in Yunnan Province, China (He et al., 2017). The variant Kawasaki 2014 emerged in several countries in Asia during winter season 2014-2015, replacing the GII.4/Sydney 2012 variant (Matsushima et al., 2015). The presence of human NoVs in NHPs raises concerns about the existence of unknown NoV reservoirs in animals with a potential for zoonotic transmission. In this study, to further investigate NoV epidemiology in NHPs, we screened a historical collection of sera obtained from seven different NHP species housed at the Zoological Garden (Bioparco) of Rome, Italy, by using an enzyme-linked immunosorbent assay (ELISA) based on virus-like particles (VLPs) generated from human NoVs of genotype GII.4 and GIV.1.

\section{2 | MATERIAL AND METHODS}

\section{1 | Serum sample collection}

A total of 86 serum samples of NHPs born in captivity were collected in the 17-year time frame spanning 2001 to 2017. The sera were obtained from 62 Japanese macaques (Macaca fuscata),

\section{Impact}

- Noroviruses (NoVs) have been identified as the most common cause of viral gastroenteritis in humans. In this study, the susceptibility to human GII and GIV NoV infections was serologically investigated in non-human primates (NHPs).

- By using an ELISA assay based on virus-like particles (VLPs) of human GII.4 and GIV.1 NoVs, specific IgG antibodies were detected in macaques, mandrills, whitecrowned mangabeys, orangutans and chimpanzees with an overall seroprevalence of $32.6 \%$.

- These findings indicate that not only GII, but also GIV strains circulate among NHPs populations. Further investigations are needed to establish firmly the possible transmission of NoVs from monkeys to humans and vice versa

10 mandrills (Mandrillus sphinx), 6 white-crowned mangabeys (Cercocebus atys lunulatus), 3 bornean orangutans (Pongo pygmaeus), 2 gorillas (Gorilla gorilla), 2 chimpanzees (Pan troglodytes) and 1 hamadryas baboon (Papio hamadryas) (Table 1). Most of the animals investigated (75/86) were adults (6-20 years old), while only 6 were older than 20 years and 5 younger than 3 years. None of the animals were specifically bled for this study, but only for regular health checks.

\section{2 | Production of virus-like particles (VLPs)}

The recombinant baculoviruses carrying the genes for the viral capsid proteins of the human NoVs, Hu/NoV/GII.4/MD14512/1987/ US and $\mathrm{Hu} / \mathrm{NoV} / \mathrm{GIV} .1 /$ SaintCloud/624/1998/US, were obtained as previously described (Bok et al., 2009; Di Martino et al., 2014, 2017). For large-scale production of VLPs, $100 \mathrm{ml}$ of Spodoptera frugiperda $(S f 9)$ cell $\left(1 \times 10^{6} \mathrm{cell} / \mathrm{ml}\right)$ suspension culture was infected with the recombinant baculovirus at a multiplicity of infection of three plaque forming units/cell. After separation from the cell debris at $48 \mathrm{hr}$ post-infection (PI), the culture medium was concentrated by ultracentrifugation through a $17 \%$ sucrose cushion in TEN-buffer (100 mM NaCl; 50 mM Tris- $\mathrm{HCl}$, pH 7.5; 1 mM EDTA) and purified on a discontinuous $20 \%-60 \%$ (wt/vol) sucrose gradient.

\begin{tabular}{llllll}
\multirow{2}{*}{$\begin{array}{l}\text { NoV virus-like } \\
\text { particles }\end{array}$} & $1: 100(\%)$ & $1: 200(\%)$ & $1: 400(\%)$ & $1: 800(\%)$ & Total (\%) \\
\cline { 2 - 6 } GII.4 & $5 / 86(5.8)$ & $3 / 86(3.5)$ & $0 / 86(0)$ & $0 / 86(0)$ & $8 / 86(9.3)$ \\
GIV.1 & $7 / 86(8.1)$ & $0 / 86(0)$ & $0 / 86(0)$ & $0 / 86(0)$ & $7 / 86(8.1)$ \\
GII.4 + GIV.1 & $5 / 86(5.8)$ & $4(4.7)$ & $2(2.3)$ & $2(2.3)$ & $13(15.1)$ \\
& $7 / 86(8.1)$ & $1(1.2)$ & $3(3.5)$ & $2(2.3)$ & \\
\hline
\end{tabular}

TABLE 1 Seroprevalence of IgG antibody against norovirus GII.4 and GIV.1 in non-human primate serum specimens

Note. GII, genogroup II; GIV, genogroup IV; NoV, norovirus. 


\subsection{Agar gel immunodiffusion (AGID)}

The reactivity between the simian IgG antibodies of the seven NHP species and the anti-human IgG antibodies was assessed by agar gel immunodiffusion (AGID) test. Agar solution consisted of $0.8 \%$ Difco $^{\text {TM }}$ agar granulated (Becton Dickson, Sparks, Maryland, USA) in Tris buffer $0.2 \mathrm{M}$ and $\mathrm{NaCl} 0.85 \%, \mathrm{pH}$ 7.2. Tests were carried out in Petri dishes using a punch of six wells of $6 \mathrm{~mm}$ in diameter and $4 \mathrm{~mm}$ apart and incubated for $72 \mathrm{hr}$ in a humid chamber at room temperature. Human anti-IgG (Sigma-Aldrich, Milan, Italy) diluted at 1:5,000 was added in the central well, while $70 \mu \mathrm{l}$ of human serum diluted 1:100 was used as positive control and $70 \mu \mathrm{l}$ of dog serum (1:100) as negative control. For each NHP species, $70 \mu \mathrm{l}$ of serum diluted at 1:100 and 1:200 was assessed.

\section{4 | Antibody-detection ELISA}

For the development of the antibody-detection ELISA, the supernatant of mock-infected Sf9 cells, NoV GII.4 and GIV.1 VLPs (final concentration of $1 \mu \mathrm{g} / \mathrm{ml}$ ) were coated onto 96 well EIA plates (Costar, Italy) at $100 \mu \mathrm{l}$ per well in carbonate-bicarbonate buffer $(0.05 \mathrm{M}, \mathrm{pH}$ 9.6), as previously described (Di Martino et al., 2014). The protein concentration of VLP preparations was determined by measuring the optical density at $280 \mathrm{~nm}\left(\mathrm{OD}_{280}\right)$ and visually by running aliquots containing bovine serum albumin (BSA) (Promega Corporation, Milan, Italy) standards on sodium dodecyl sulphate-10\% polyacrylamide gel electrophoresis. The plates were incubated at $4^{\circ} \mathrm{C}$ overnight. The wells were washed five times with $0.1 \%$ Tween-phosphatebuffered saline (PBS-T) and then blocked with $300 \mu \mathrm{l}$ of Superblock (Invitrogen, Ltd, Milan, Italy) at room temperature for $5 \mathrm{~min}$. After washing five times with PBS-T, each NHPs serum sample $(100 \mu \mathrm{l})$ diluted 1:100 in Superblock (Invitrogen, Ltd, Milan, Italy) was added to the antigen-coated wells and the plates were incubated at $37^{\circ} \mathrm{C}$ for $1 \mathrm{hr}$. After incubation with horseradish peroxidase-conjugated goat anti-human IgG (Sigma-Aldrich, Milan, Italy) at 1:5,000 dilution for $30 \mathrm{~min}$ at $37^{\circ} \mathrm{C}$, the reaction was developed with the addition of 2,20-azino-di-(3-ethylbenzthiazoline-6-sulphonate) (ABTS) (Invitrogen, Ltd, Milan, Italy) substrate and the incubation at room temperature for $12 \mathrm{~min}$. Absorbance was measured at $405 \mathrm{~nm}$ using a multiskan automatic plate reader (Thermo Labsystems, Finland).
The cut-off point of the ELISA $\left(\mathrm{OD}_{405} \geq 0.5\right)$ was established as the mean of the $\mathrm{OD}_{405}$ readings of $25 \mathrm{NHP}$ serum samples negative in western blotting for GII.4 and GIV.1 VLPs plus 2 standard deviations. For each tested sample, a positive/negative ratio $\left(\mathrm{OD}_{405}\right.$ of VLPs/ $\mathrm{OD}_{405}$ of mock-infected cells) $\geq 2.0$ was used to evaluate the background binding. All samples that had $\mathrm{OD}_{405}$ values $\geq 0.5$ at the initial dilution of 1:100 were considered positive and titrated in 2-fold dilutions. Mean ELISA antibody titres were calculated and expressed as the reciprocal of the highest serum dilution that had positive absorbance $\left(\mathrm{OD}_{405} \geq 0.5\right)$ for GII.4 and/or GIV.1 antigens.

\subsection{Statistical analysis}

Statistical analysis was performed using GraphPad Prism Software (https://www.graphpad.com/scientific-software/prism/). Pearson's rank correlation test and paired sample $t$ test were applied to assess the correlation between the two NoV-VLPs serum titres. Fisher's exact test was used to determine the difference in seroprevalence rates among the seven NHPs species and the difference among the age groups. A $p$ value of $<0.05$ was considered statistically significant.

\section{3 | RESULTS}

A total of 86 serum samples collected from seven NHPs species housed at the Zoological Garden of Rome (Italy) were screened for the presence of antibodies against human GII.4 and GIV.1 NoVs.

Firstly, to ascertain the cross-reactivity between the simian IgG antibodies and the anti-human IgG antibodies, AGID test was performed for each NHP species investigated. The results obtained demonstrated that the serum of each monkey species was reactive either at dilution of 1:100 or 1:200 (Figure 1). When the samples were analysed by using the recombinant ELISA, out of 86 NHP serum samples tested at the initial dilution of $1: 100,32.6 \%$ (28/86) reacted with at least one NoV antigen. In detail, 8 (9.3\%) sera reacted only with GII.4, 7 (8.1\%) only with GIV.1 and a total of $13(15.1 \%)$ reacted with both GII.4 and GIV.1 VLPs. When all the positive sera were rescreened by endpoint titration, 5 (5.8\%) samples reacted with the GII.4 antigen at dilution of $1: 100$ and 3 (3.5\%) at 1:200. Seven (8.1\%) samples reacted with GIV.1 VLPs at
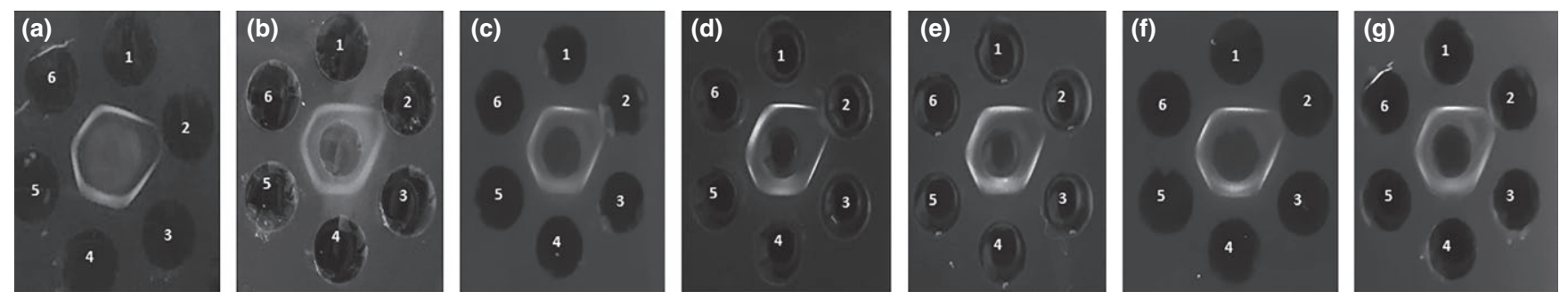

FIGURE 1 Agar gel immunodiffusion of each NHP species IgG antibodies and the anti-human IgG antibodies (central well). Peripheral wells contain a human serum as positive control (wells 1, 3, 5), a dog serum as negative control (well 2) and a NHP serum diluted 1:100 (well 4) and 1:200 (well 6). The precipitation lines (arrows) indicate the cross-reactivity between NHP IgG and anti-human IgG. (a) Japanese macaques; (b) white-crowned mangabey; (c) mandrill; (d) gorilla; (e) bornean orangutan; (f) chimpanzee; (g) hamadryas baboon 
dilution of $1: 100$. Of 13 sera positive against both the antigens, eight sera (6.9\%) reacted either with GII.4 or GIV.1 at the final dilution of $1: 100(4.6 \%, 4 / 86), 1: 400(2.3 \%, 2 / 86)$ and $1: 800(2.3 \%$, $2 / 86)$, respectively. Three samples (3.5\%) reacted at a dilution of 1:200 with GII.4 and at 1:100 with GIV.1, one serum at a dilution of 1:200 with GII.4 and 1:400 with GIV.1 and an additional serum sample reacted at a dilution of 1:100 with GII.4 and 1:200 with GIV.1 (Table 1). Analyses performed by Pearson's rank and $t$ test revealed a high correlation between the levels of antibodies to GII.4 and GIV.1 antigens ( $p<0.0001 ; r=0.907$ ).

Among the seven NHP species investigated in this study, IgG antibodies against GII.4 NoVs were found in Japanese macaques (29.0\%, 18/62), white-crowned mangabey $(16.6 \%, 1 / 6)$, orangutan $(33.3 \%, 1 / 3)$ and mandrill $(10.0 \%, 1 / 10)$. Twelve macaques $(19.3 \%$, 12/62), 5 mandrills (50.0\%, 5/10), 2 chimpanzees (100\%, 2/2) and a white-crowned mangabey $(16.6 \%, 1 / 6)$ showed positivity for NoV GIV.1 VLPs. IgG antibodies against the two NoV antigens were not detected in gorillas and hamadryads (Table 2). Considering the seropositivity by year of sample collection (Figure 2 ), the overall prevalence ranged from $15.0 \%(3 / 20)$ in 2001 , to $37.5 \%(3 / 8)$ in 2002 , to $14.3 \%(1 / 7)$ in 2004 , to $66.6 \%(2 / 3)$ in 2005 , to $80.0 \%$ $(4 / 5)$ in 2006 , to $50.0 \%(1 / 2)$ in 2007 and 2008, respectively, and to $40.0 \%(2 / 5)$ in 2009 . A high seroprevalence $(44.0 \%, 11 / 25)$ was observed in the sera collected only from macaques in 2010. Of these, 4 animals were positive for GII.4, 1 only for GIV.1 and 6 either for GII.4 or GIV.1. None of the animals assessed in 2003 (3) and during 2011-2017 (6) were positive for IgG antibodies against the two NoV antigens.

\section{4 | DISCUSSION}

ELISAs based on VLPs have successfully been used to gather information on the epidemiology of NoVs in humans and animals ( $\mathrm{Di}$ Martino et al., 2014, 2017). In this study, using baculovirus-generated
VLPs based on human GII.4 and GIV.1 NoVs, IgG-specific antibodies were detected in Japanese macaques, mandrills, chimpanzees, white-crowned mangabey and orangutan. These findings confirm that captive NHPs are susceptible to infections by GII NoVs. In addition, this is the first study investigating the seroprevalence of antiGIV.1 antibodies in monkeys. Human NoV GIV.1 (Alphatron-like) has been identified only sporadically in human patients (Ao, Yu, Li, Jin, \& Duan, 2014; La Rosa, Pourshaban, laconelli, \& Muscillo, 2008; Muscillo et al., 2013), although epidemiological studies based on the analysis of sewage and wastewater in Japan and Italy have revealed, unexpectedly, prevalence rates as high as $21.4 \%$ and $21.8 \%$, respectively (Kitajima, Haramoto, Phanuwan, Katayama, \& Ohgaki, 2009; Muscillo et al., 2013). A study conducted on an age-stratified collection of human serum samples (Di Martino et al., 2014) has revealed a seroprevalence for GIV.1 of $22.0 \%$, thus suggesting that these NoVs are more common in human populations than previously believed. Susceptibility of NHPs to GIV NoV infections was also suspected in a molecular survey in macaques (Farkas, 2016). By using a quantitative RT-PCR, the RNA of GIV NoVs was detected in $0.6 \%(3 / 500)$ macaque faecal samples, although sequence information for the GIV-positive samples was not obtained (Farkas, 2016).

In our analysis, IgG antibodies against GII.4 and GIV.1 NoVs were found, respectively, in $24.4 \%$ (21/86) and $23.2 \%$ (20/86) of the sera assessed, suggesting frequent exposure of NHPs to these viruses. For thirteen positive samples, reactivity against both GII.4 and GIV.1 was revealed with titres ranging from 1:100 to 1:800. Previous evidence demonstrated that GI (GI.1, GI.2, GI.3) and GII (GII.4, GII.6, GII.12) VLPs are antigenically unrelated to GIV.1 and GIV.2 NoVs (Caddy et al., 2015; Di Martino et al., 2014), while cross-reactivity has been observed between GII.4 and GVI.2 genotypes (Di Martino et al., 2017), as well as between GIV.1 and GIV.2 (Di Martino et al., 2014). Accordingly, it could be argued that the dual reactivity for GII.4 and GIV.1 antigens found in 13 NHP sera was the result of a coinfection with GII.4 and GIV.1 NoVs or sequential infections over time. Otherwise, we cannot rule out the possibility that monkeys

\begin{tabular}{|c|c|c|c|c|c|}
\hline NHPs tested & $\begin{array}{l}\text { Positive } \\
\text { Gll.4 IgG }\end{array}$ & $\begin{array}{l}\text { Negative } \\
\text { Gll.4 IgG }\end{array}$ & $\begin{array}{l}\text { Positive } \\
\text { GIV.1 IgG }\end{array}$ & $\begin{array}{l}\text { Negative } \\
\text { GIV.1 IgG }\end{array}$ & Tota \\
\hline $\begin{array}{l}\text { Macaca fuscata (Japanese } \\
\text { macaque) }\end{array}$ & $18(29.0 \%)$ & 44 (70.9\%) & 12 (19.3\%) & $50(80.6 \%)$ & 62 \\
\hline $\begin{array}{l}\text { Mandrillus sphinx } \\
\text { (Mandrill) }\end{array}$ & $1(10.0 \%)$ & 9 (90.0\%) & $5(50.0 \%)$ & $5(50.0 \%)$ & 10 \\
\hline $\begin{array}{l}\text { Cercocebus atys lunulatus } \\
\text { (White-crowned } \\
\text { mangabey) }\end{array}$ & $1(16.6 \%)$ & 5 (83.3\%) & $1(16.6 \%)$ & $5(16.6 \%)$ & 6 \\
\hline $\begin{array}{l}\text { Pongo pygmaeus (Bornean } \\
\text { orangutan) }\end{array}$ & 1 (33.3\%) & $2(66.6 \%)$ & $0(0.0 \%)$ & $3(100 \%)$ & 3 \\
\hline Gorilla gorilla (Gorilla) & $0(0.0 \%)$ & 2 (100\%) & $0(0.0 \%)$ & 2 (100\%) & 2 \\
\hline $\begin{array}{r}\text { Pan troglodytes } \\
\text { (Chimpanzee) }\end{array}$ & $0(0.0 \%)$ & $2(100 \%)$ & 2 (100.0\%) & 0 (0.0\%) & 2 \\
\hline $\begin{array}{l}\text { Papio hamadryas } \\
\text { (Hamadryas baboon) }\end{array}$ & 0 (0.0\%) & $1(100 \%)$ & $0(0.0 \%)$ & 1 (100\%) & 1 \\
\hline
\end{tabular}

TABLE 2 NHP species assessed for the presence of IgG antibodies against NoV GII.4 and GIV.1

Note. GII, genogroup II; GIV, genogroup IV; NHP, non-human primate; NoV, norovirus. 


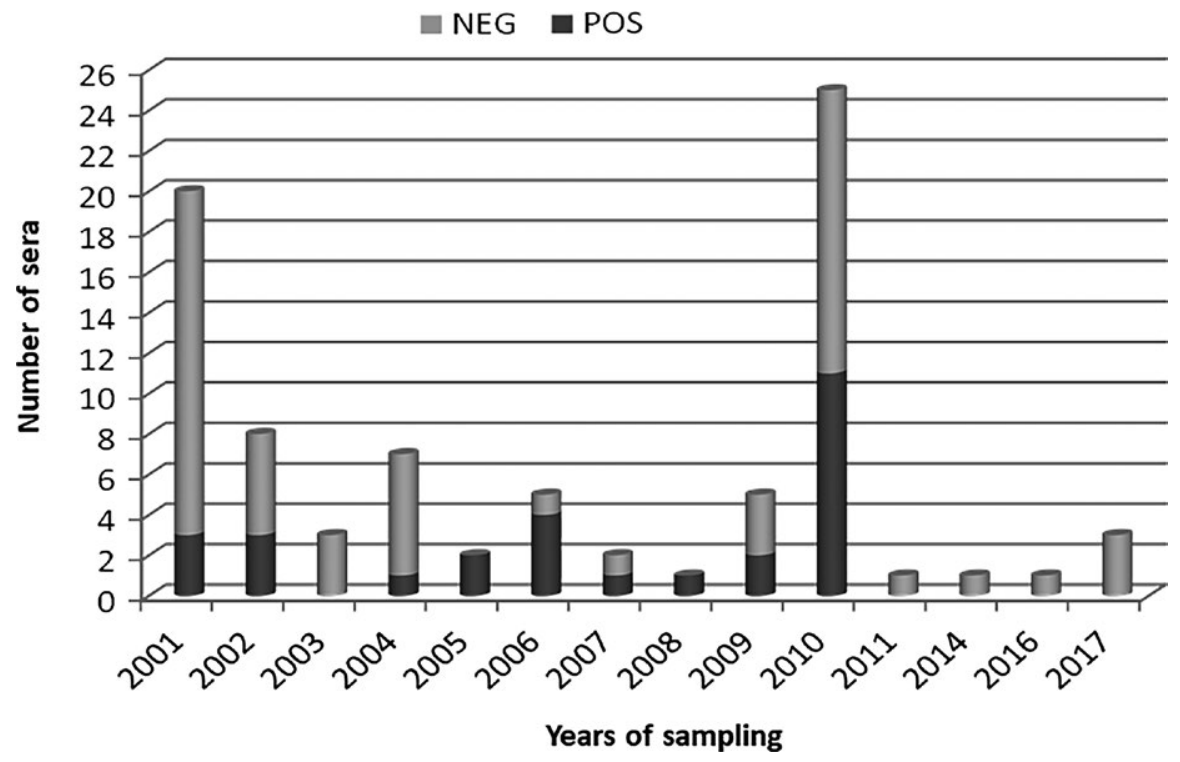

FIGURE 2 Seropositivity against NoVs in NHPs in the 17-year time frame spanning 2001 to 2017

\section{Years of sampling}

time span. Finally, we cannot rule out the circulation of strains unrelated antigenically to the NoV antigens used in our assay.

In conclusion, our findings demonstrate that not only GII, but also GIV strains circulate widely among NHPs populations. Whether and to which extent transmission of NoVs from monkeys to humans and vice versa occurs under natural and captive conditions should be assessed, to understand the ecology and evolution of human NoVs and track the origin of novel NoV strains.

\section{ACKNOWLEDGEMENTS}

This study was supported by grants from the University of Teramo, Italy, and from the Italian Ministry of University. Sandra Bermudez Sanchez is granted by Marie Skłodowska-Curie COFUNDREP-EAT 713714: Innovative Research and Training Doctoral Programme in the interdisciplinary domain of food and healthy diet.

\section{CONFLICT OF INTEREST}

All authors declare that there are no financial or other relationships that might lead to a conflict of interest. All authors have seen and approved the manuscript and have contributed significantly to the work.

\section{ORCID}

Barbara Di Martino (iD https://orcid.org/0000-0001-6192-7166

\section{REFERENCES}

Ao, Y. Y., Yu, J. M., Li, L. L., Jin, M., \& Duan, Z. J. (2014). Detection of human norovirus GIV.1 in China: A case report. Journal of Clinical Virology, 61(2), 298-301. https://doi.org/10.1016/j.jcv.2014.08.002

Bok, K., Abente, E. J., Realpe-Quintero, M., Mitra, T., Sosnovtsev, S. V., Kapikian, A. Z., \& Green, K. Y. (2009). Evolutionary dynamics of GII.4 
noroviruses over a 34-year period. Journal of Virology, 83(22), 1189011901. https://doi.org/10.1128/JVI.00864-09

Bok, K., Parra, G. I., Mitra, T., Abente, E., Shaver, C. K., Boon, D., ... Green, K. Y. (2011). Chimpanzees as an animal model for human norovirus infection and vaccine development. Proceedings of the National Academy of Sciences of the United States of America, 108(1), 325-330. https://doi.org/10.1073/pnas.1014577107

Caddy, S. L., De Rougemont, A., Emmott, E., El-Attar, L., Mitchell, J. A., Hollinshead, M., ... Goodfellow, I. (2015). Evidence for human norovirus infection of dogs in the United Kingdom. Journal of Clinical Microbiology, 53(6), 1873-1883. https://doi.org/10.1128/ JCM.02778-14

Di Martino, B., Di Profio, F., Ceci, C., Di Felice, E., Green, K. Y., Bok, K., ... Martella, V. (2014). Seroprevalence of norovirus genogroup IV antibodies among humans, Italy, 2010-2011. Emerging Infectious Diseases, 20(11), 1828-1832. https://doi.org/10.3201/eid2011.131601

Di Martino, B., Di Profio, F., Melegari, I., Sarchese, V., Massirio, I., Palermo, G., ... Martella, V. (2017). Seroprevalence for norovirus genogroup II, IV and VI in dogs. Veterinary Microbiology, 203, 68-72. https://doi. org/10.1016/j.vetmic.2017.03.006

Farkas, T. (2016). Natural norovirus infections in Rhesus Macaques. Emerging Infectious Diseases, 22(7), 1272-1274. https://doi. org/10.3201/eid2207.151740

Farkas, T., Cross, R. W., Hargitt, E., Lerche, N. W., Morrow, A. L., \& Sestak, K. (2010). Genetic diversity and histo-blood group antigen interactions of rhesus enteric caliciviruses. Journal of Virology, 84(17), 8617-8625. https://doi.org/10.1128/JVI.00630-10

Farkas, T., Dufour, J., Jiang, X., \& Sestak, K. (2010). Detection of norovirus-, sapovirus- and rhesus enteric calicivirus-specific antibodies in captive juvenile macaques. The Journal of General Virology, 91(Pt 3), 734-738. https://doi.org/10.1099/vir.0.015263-0

Green, K. (2013). Caliciviridae: the noroviruses. In D. M. Knipe, P. M. Howley, J. I. Cohen, D. E. Griffin, R. A. Lamb, M. A. Martin, \& B. Roizman (Eds.), Fields virology (6th ed., pp. 583-609). Philadelphia, PA: Lippincott Williams \& Wilkins.

He, Z., Liu, B., Tao, Y., Li, C., Xia, M., Zhong, W., ... Tan, M. (2017). Norovirus GII.17 natural infections in Rhesus Monkeys, China. Emerging Infectious Diseases, 23(2), 316-319. https://doi.org/10.3201/ eid2302.161077

Jiang, B., McClure, H. M., Fankhauser, R. L., Monroe, S. S., \& Glass, R. I. (2004). Prevalence of rotavirus and norovirus antibodies in nonhuman primates. Journal of Medical Primatology, 33(1), 30-33. https:// doi.org/10.1111/j.1600-0684.2003.00051.x

Kitajima, M., Haramoto, E., Phanuwan, C., Katayama, H., \& Ohgaki, S. (2009). Detection of genogroup IV norovirus in wastewater and river water in Japan. Letters in Applied Microbiology, 49(5), 655-658. https://doi.org/10.1111/j.1472-765X.2009.02718.x

La Rosa, G., Pourshaban, M., laconelli, M., \& Muscillo, M. (2008). Detection of genogroup IV noroviruses in environmental and clinical samples and partial sequencing through rapid amplification of cDNA ends. Archives of Virology, 153(11), 2077-2083. https://doi. org/10.1007/s00705-008-0241-4

Li, X., Zhou, R., Tian, X., Li, H., \& Zhou, Z. (2010). Characterization of a cross-reactive monoclonal antibody against Norovirus genogroups I, II, III and V. Virus Research, 151(2), 142-147. https://doi.org/10.1016/j. virusres.2010.04.005
Lopman, B. A., Steele, D., Kirkwood, C. D., \& Parashar, U. D. (2016). The vast and varied global burden of norovirus: prospects for prevention and control. PLoS Medicine, 13(4), e1001999. https://doi. org/10.1371/journal.pmed.1001999

Matsushima, Y., Ishikawa, M., Shimizu, T., Komane, A., Kasuo, S., Shinohara, M., ... Shimizu, H. (2015). Genetic analyses of GII.17 norovirus strains in diarrheal disease outbreaks from December 2014 to March 2015 in Japan reveal a novel polymerase sequence and amino acid substitutions in the capsid region. Euro Surveillance: Bulletin Européen Sur Les Maladies Transmissibles = European Communicable Disease Bulletin, 20(26), 21173.

Muscillo, M., Fratini, M., Graffeo, R., Sanguinetti, M., Martella, V., Green, K. Y., \& La Rosa, G. (2013). GIV noroviruses in wastewaters and in stool specimens from hospitalized patients. Food and Environmental Virology, 5, 194-202. https://doi.org/10.1007/s12560-013-9121-5

Parker, T. D., Kitamoto, N., Tanaka, T., Hutson, A. M., \& Estes, M. K. (2005). Identification of Genogroup I and Genogroup II broadly reactive epitopes on the norovirus capsid. Journal of Virology, 79(12), 7402-7409. https://doi.org/10.1128/JVI.79.12.7402-7409.2005

Parra, G. I., Azure, J., Fischer, R., Bok, K., Sandoval-Jaime, C., Sosnovtsev, S. V., ... Green, K. Y. (2013). Identification of a broadly cross-reactive epitope in the inner shell of the norovirus capsid. PLoS One, 8, e67592.

Patel, M. M., Widdowson, M. A., Glass, R. I., Akazawa, K., Vinjé, J., \& Parashar, U. D. (2008). Systematic literature review of role of noroviruses in sporadic gastroenteritis. Emerging Infectious Diseases, 14(8), 1224-1231. https://doi.org/10.3201/eid1408.071114

Rockx, B. H., Bogers, W. M., Heeney, J. L., Van Amerongen, G., \& Koopmans, M. P. (2005). Experimental norovirus infections in nonhuman primates. Journal of Medical Virology, 75(2), 313-320. https:// doi.org/10.1002/jmv.20273

Sestak, K., Feely, S., Fey, B., Dufour, J., Hargitt, E., Alvarez, X., ... Farkas, T. (2012). Experimental inoculation of juvenile rhesus macaques with primate enteric caliciviruses. PLoS ONE, 7(5), e37973. https://doi. org/10.1371/journal.pone.0037973

Subekti, D. S., Tjaniadi, P., Lesmana, M., McArdle, J., Iskandriati, D., Budiarsa, I. N., ... Oyofo, B. A. (2002). Experimental infection of Macaca nemestrina with a Toronto Norwalk-like virus of epidemic viral gastroenteritis. Journal of Medical Virology, 66(3), 400-406. https://doi.org/10.1002/jmv.2159

Vinjé, J. (2015). Advances in laboratory methods for detection and typing of norovirus. Journal of Clinical Microbiology, 53(2), 373-381. https:// doi.org/10.1128/JCM.01535-14

Wyatt, R. G., Greenberg, H. B., Dalgard, D. W., Allen, W. P., Sly, D. L., Thornhill, T. S., ... Kapikian, A. Z. (1978). Experimental infection of chimpanzees with the Norwalk agent of epidemic viral gastroenteritis. Journal of Medical Virology, 2(2), 89-96. https://doi.org/10.1002/ jmv.1890020203

How to cite this article: Di Profio F, Sarchese V, Melegari I, et al. Seroprevalence for norovirus genogroups GII and GIV in captive non-human primates. Zoonoses Public Health. 2019;00:1-6. https://doi.org/10.1111/zph.12566 